\title{
DIFFUSE X-RAY EMISSION IN SPIRAL GALAXIES
}

\author{
Krystal Tyler, ${ }^{1,2}$ A. C. Quillen, ${ }^{1}$ Amanda LaPage, ${ }^{1}$ and George H. Rieke ${ }^{3}$ \\ Received 2003 August 6; accepted 2004 March 29
}

\begin{abstract}
We compare the soft diffuse X-ray emission from Chandra images of 12 nearby intermediate-inclination spiral galaxies to the morphology seen in $\mathrm{H} \alpha$, molecular gas, and mid-infrared emission. We find that diffuse X-ray emission is often located along spiral arms in the outer parts of spiral galaxies but tends to be distributed in a more nearly radially symmetric morphology in the center. The X-ray morphology in the spiral arms matches that seen in the mid-infrared or $\mathrm{H} \alpha$ and thus implies that the X-ray emission is associated with recent active star formation. In the spiral arms there is a good correlation between the level of diffuse X-ray emission and that in the mid-infrared in different regions. The correlation between X-ray and mid-IR flux in the galaxy centers is less strong. We also find that the central X-ray emission tends to be more luminous in galaxies with brighter bulges, suggesting that more than one process is contributing to the level of central diffuse X-ray emission. We see no strong evidence for X-ray emission trailing the location of high-mass star formation in spiral arms. However, population synthesis models predict a high mechanical energy output rate from supernovae for a time period that is about 10 times longer than the lifetime of massive ionizing stars, conflicting with the narrow appearance of the arms in X-rays. The fraction of supernova energy that goes into heating the interstellar medium must depend on environment and is probably higher near sites of active star formation. The X-ray estimated emission measures suggest that the volume filling factors and scale heights are low in the outer parts of these galaxies but higher in the galaxy centers. The differences between the X-ray properties and morphology in the centers and outer parts of these galaxies suggest that galactic fountains operate in outer galaxy disks but that winds are primarily driven from galaxy centers.
\end{abstract}

Subject headings: galaxies: ISM — galaxies: spiral — X-rays: galaxies

\section{INTRODUCTION}

Results from both Einstein and ROSAT have shown that spiral galaxies emit soft X-rays $(0.1-2.4 \mathrm{keV})$ with luminosities of $10^{38}-10^{40}$ ergs s$^{-1}$ (Fabbiano et al. 1992; Read et al. 1997; Cui et al. 1996). Before the launch of Chandra, the study of the diffuse X-ray emission from spiral galaxies was seriously impeded by lack of spatial resolution. Point sources could not be well separated from the diffuse thermal gas, making it difficult to compare the morphology of the X-ray emission to that in other bands. Study of the morphology of diffuse emission from the disks of spiral galaxies was therefore possible only for some nearby galaxies (e.g., M101, Snowden \& Pietsch 1995; M51, Ehle et al. 1995; NGC 891, Bregman \& Houck 1997).

The spatial resolution of Chandra not only allows separation between diffuse emission and point sources but also allows a more precise morphological study of the diffuse emission. Among normal galaxies, recent Chandra imaging has shown that the diffuse emission can have different types of morphology: X-ray-emitting gas above the plane of the galaxy that has been interpreted to be caused by winds escaping the galaxy due to mechanical energy output from massive stars and supernovae (e.g., Strickland et al. 2004b; Wang et al. 2001); coronal X-ray-emitting gas associated with galactic fountains (e.g., Fraternali et al. 2002 in NGC 2403; Kuntz et al. 2003 in M101). The diffuse hot gas is due to thermal

\footnotetext{
${ }^{1}$ Department of Physics and Astronomy, University of Rochester, Rochester,NY14627;ktyler@physics.purdue.edu, aquillen@pas.rochester.edu, al007m@mail.rochester.edu.

2 Purdue University, Department of Physics, 525 Northwestern Avenue, West Lafayette, IN 47907.

3 Steward Observatory, University of Arizona, Tucson, AZ 85721; grieke@ as.arizona.edu.
}

emission from a fairly cool $k T \sim 0.2 \mathrm{keV}\left(T \sim 10^{6} \mathrm{~K}\right)$ component (Read et al. 1997; Strickland et al. 2004a).

The gas detected in X-rays is suspected to be associated with supernovae and winds from young massive stars. Since supernovae generate metals, the transport of this hot gas is key toward understanding the metallicity evolution of galaxies (e.g., Edmunds 1990). Superwinds are of cosmological interest as they can transport large amounts of gas, in particular newly synthesized heavy elements, and energy into the intergalactic medium (IGM). However, if this gas cannot escape from the disk into the halo region, it will fall down onto the disk. In a galactic fountain model, the hot gas escaping from the disk cools quickly because of adiabatic expansion. Anomalous high-velocity $\mathrm{H}$ i clouds are suspected to represent such an infalling phase of the galactic fountain, whereas the hot $\mathrm{X}$-ray-emitting gas would pertain to the initial outgoing phase of it (Bregman 1980; de Avillez 2000; Norman \& Ikeuchi 1989). When the star formation rate is sufficiently high, largescale energetic outflows are predicted (Chevalier \& Clegg 1985; Heckman et al. 1990). These "superwinds" may account for the X-ray emission seen well above the planes of edge-on starburst galaxies and are potentially capable of enriching and heating the IGM (e.g., Martin et al. 2002).

The morphology and quantity of the hot X-ray-emitting gas is therefore related to the general problem of understanding the scales (spatial and temporal) over which metal enrichment takes place in spiral galaxies. X-ray emission associated with spiral structure should trace local, relatively short timescale, localized metallicity enrichment due to the movement of the spiral density wave, whereas larger scale superwinds are more likely to be relevant to the enrichment of the IGM.

Many Chandra observing programs have focused their studies on individual galaxies or on the populations of 
TABLE 1

Archived Nearby Galaxies with Diffuse X-Ray Emission

\begin{tabular}{|c|c|c|c|c|}
\hline Galaxy & X-Ray Morphology & Observation ID & Nuclear Type & $\begin{array}{c}\text { Distance } \\
(\mathrm{Mpc})\end{array}$ \\
\hline M51 …......................... & Arms, center & 1622 & Sy2 & 7.7 \\
\hline M83 ....... & Arms, center & 793 & $\mathrm{H} 2$ & 4.5 \\
\hline M101 …........................ & Arms, center & 934 & & 7.4 \\
\hline NGC $2403 \ldots \ldots \ldots \ldots \ldots \ldots$ & Irregular & 2014 & $\mathrm{H} 2$ & 4.2 \\
\hline 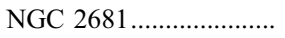 & Center & 2060 & L1.9 & 13.3 \\
\hline NGC $3184 \ldots$. & Arms, center & 804,1520 & $\mathrm{H} 2$ & 8.7 \\
\hline 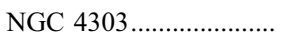 & Arms, center & 2149 & $\mathrm{H} 2$ & 15.2 \\
\hline NGC 4579 .. & Center & 807 & S1.9/L1.9 & 16.8 \\
\hline NGC $4736 \ldots \ldots \ldots \ldots \ldots \ldots \ldots$ & Arms, center & 808 & L2 & 4.3 \\
\hline NGC $5055 \ldots \ldots \ldots \ldots \ldots \ldots \ldots$ & Center & 2197 & $\mathrm{~T} 2$ & 7.2 \\
\hline 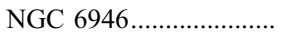 & Arms,center & 1043 & $\mathrm{H} 2$ & 5.5 \\
\hline NGC $7331 \ldots \ldots \ldots \ldots \ldots \ldots \ldots$ & Center & 2198 & $\mathrm{~T} 2$ & 15.1 \\
\hline
\end{tabular}

Notes.-Observation IDs are those listed by the Chandra X-ray Center. Nuclear types and distances are those compiled by Helfer et al. (2003), except for the distance to M83, which was measured by Thim et al. (2003), and that to NGC 2681 from Tully (1988) assuming a Hubble constant of $75 \mathrm{Mpc}^{-1} \mathrm{~km} \mathrm{~s}^{-1}$.

unresolved sources in these galaxies. Only in the last year have a sufficient number of deep Chandra images of galaxies become available, enabling us to carry out a comparative study of the diffuse components. The recent study of Strickland et al. (2004a, 2004b) focuses on edge-on galaxies. Here we carry out a complementary study by focusing on low- or intermediateinclination galaxies. These galaxies allow us to compare the X-ray morphology to the distribution of stellar components (bulge and spiral arms) and sites of active star formation.

In $\S 2$ we describe the sample of galaxies and our procedure for producing images that highlight the diffuse component. We also describe comparison data used to probe for correlations between galactic components. In $\S 3$ we compare X-ray fluxes and morphology to those observed at other wavelengths. A summary and discussion follows in $\S 4$.

\section{OBSERVATIONS}

\subsection{The Sample}

We searched the Chandra X-ray archive for intermediate- or low-inclination nearby galaxies, observed in imaging modes with ACIS-S at exposure times that are longer than $20 \mathrm{ks}$. Shorter exposures are not capable of detecting sufficient numbers of photons from a diffuse component to enable a good study of its morphology. We found 12 galaxies that met our criteria; they are listed in Table 1, along with additional descriptive information. NGC 1068 and NGC 4258 were omitted from the sample because of their bright Seyfert nuclei.

\subsection{Diffuse X-Ray Emission Maps}

Reduction of Chandra ACIS-S images was done with CIAO version 2.3. ${ }^{4}$ Light curves were constructed from the entire S3 chip to allow detection and removal of background flares. Because the diffuse emission component is soft, we removed photons with estimated energies greater than $1.5 \mathrm{keV}$. This high-energy cut increased our ability to detect the diffuse component against hard background events, which were possibly from low-level flares that were not detected from the light curves. To ensure that we were justified in making such an

\footnotetext{
${ }^{4}$ CIAO Version 2.3, http://cxc.harvard.edu/ciao2.3science threads can be found at http://cxc.harvard.edu/ciao2.3/threads/index.html.
}

energy cut, we made color maps of all galaxies with respect to the estimated photon energy. The color maps were split into red $(0.3-1.5 \mathrm{keV})$, green $(1.5-2.5 \mathrm{keV})$, and blue $(2.5-$ $8.0 \mathrm{keV})$. The background contained points of all three colors, while the diffuse X-ray emission from the galaxies was mostly red, confirming that it is soft.

Point sources were identified from the Chandra event files by masking elliptical areas around each source. Each region was identified by the CIAO routine wavdetect and was then visually inspected to enlarge, if necessary, the masking area or to determine if diffuse emission had been misidentified as point sources, especially in crowded regions. Pixels inside the ellipses were filled in based on local background values using the CIAO routine dmfilth. We used the DIST method to fill in the background because the count rates were not sufficiently high to use the POISSON method. ${ }^{5}$ After point sources were removed, we smoothed the images using the adaptive routine, csmooth, with a maximum smoothing length of 20 pixels. Images were corrected for the exposure time and effective telescope area using the CIAO routine mergeall to build the final fluxed images that are shown in Figure 1. The smoothed images shown in Figure 1 were used for morphological comparisons. However, to measure fluxes from particular regions, we used the unsmoothed images.

\subsection{Comparison Images}

To identify what processes are affecting the distribution of X-ray gas, we searched for images of our sample galaxies at other wavelengths. Many of these galaxies were also observed in $\mathrm{CO}(1-0)$ tracing the molecular gas component as part of the BIMA-SONG survey (Helfer et al. 2003). We also downloaded near-infrared $H$-band images tracing the stellar component from the Two Micron All Sky Survey (2MASS). We searched NED for narrowband images of the $\mathrm{H} \alpha$ emission line and mid-infrared images at 12 or $15 \mu \mathrm{m}$ from the Infrared

\footnotetext{
${ }^{5}$ For the DIST method, pixel values in the source regions are sampled from the distribution of pixel values in the background regions. For the POISSON method, pixel values in the source regions are sampled from the Poisson distribution whose mean is that of the pixel values in the background region. See http://asc.harvard.edu/ciao/ahelp/dmfilth.html.
} 

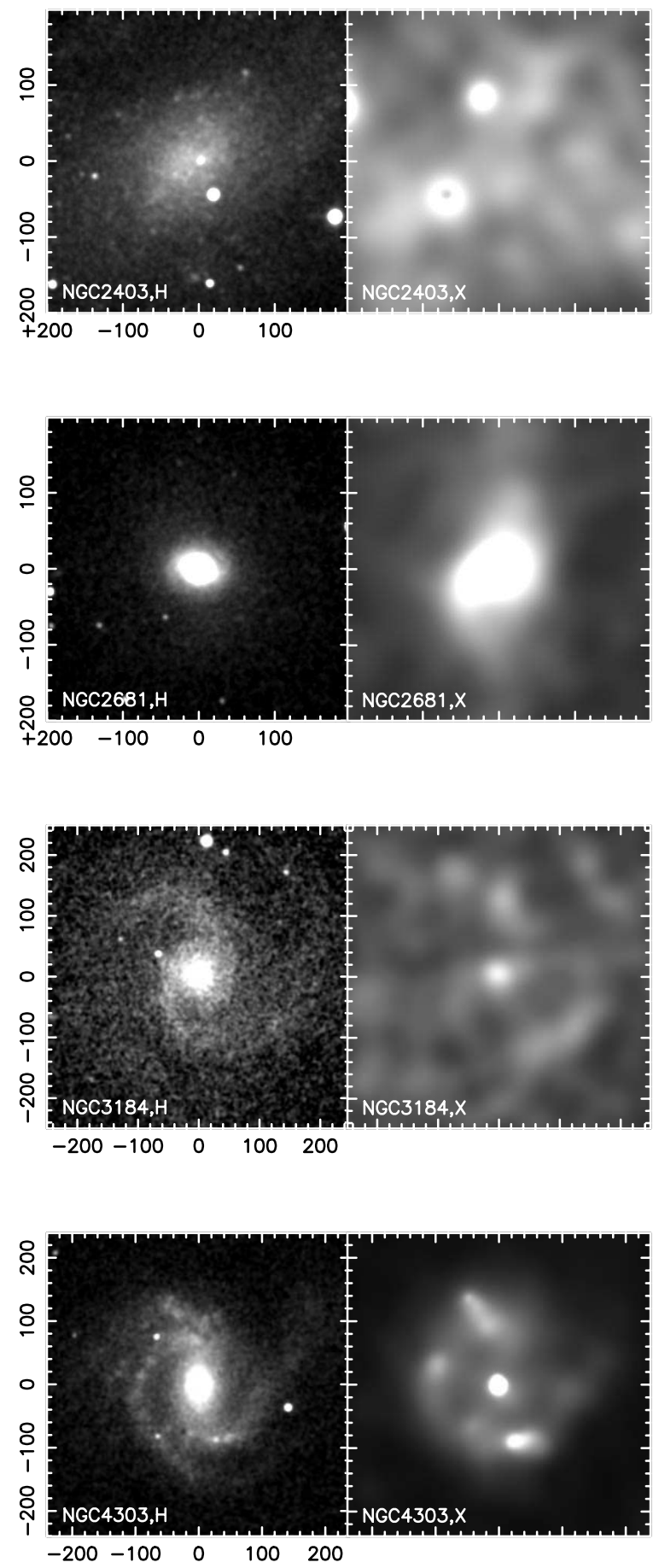

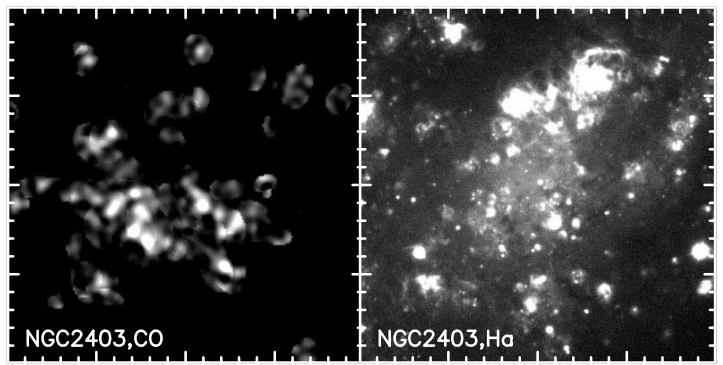

FIG. $1 a$

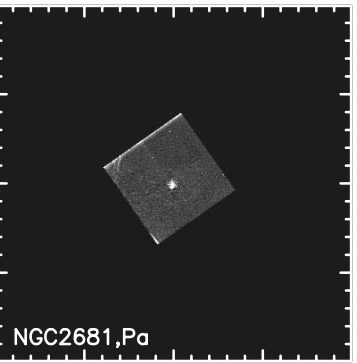

FIG. $1 b$

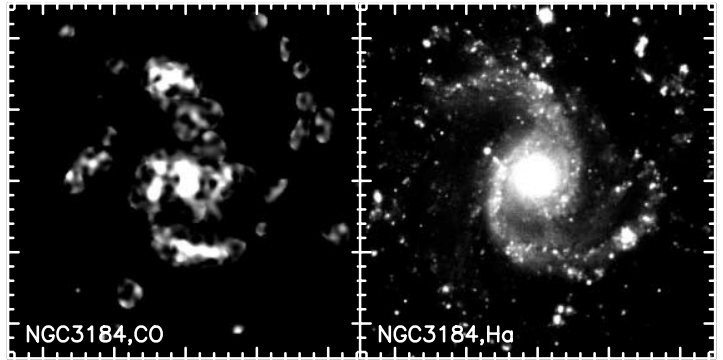

Fig. $1 c$

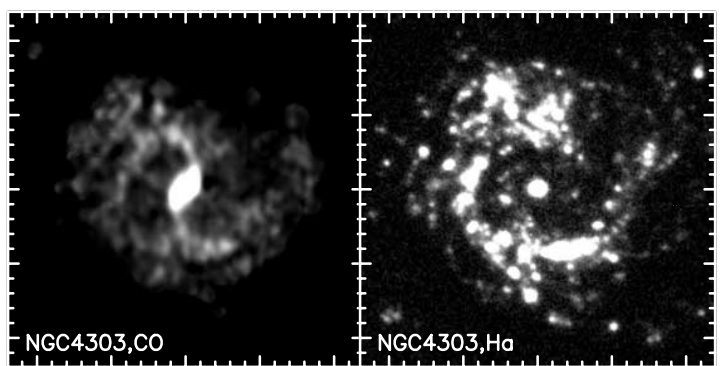

FIG. $1 d$
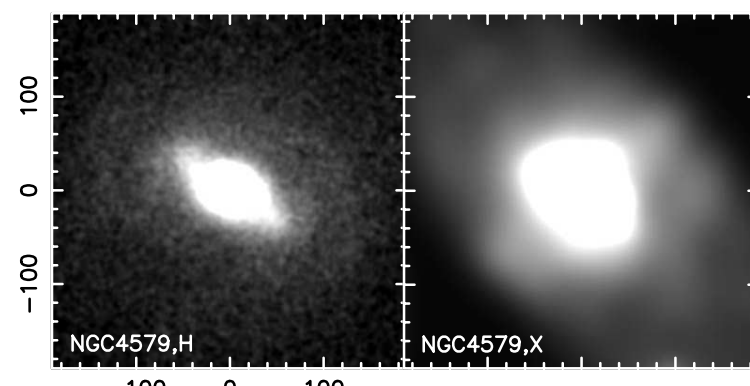

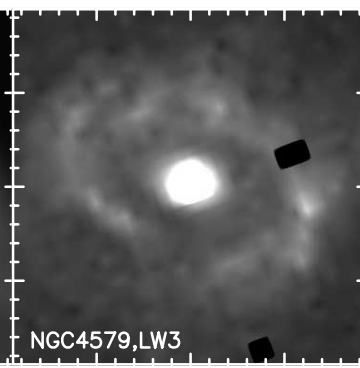

FIG. 1e

FIG. 1.-Diffuse X-ray emission compared to other galactic tracers. From left to right in each panel we show the 2MASS $H$-band tracing starlight, the diffuse X-ray emission from the Chandra ACIS-S image, 12 or $15 \mu \mathrm{m}$ ISOCAM images showing hot dust, the molecular gas as seen in CO emission, and a narrowband $\mathrm{H} \alpha$ image tracing star formation. North is up, and west is to the right. Axes are given in arcseconds from the galaxy nucleus. A Pa $\alpha$ image is shown instead of H $\alpha$ for NGC 2681 and NGC 7331. When there is no panel, we did not obtain an image in that wavelength band. (a) NGC 2403. (b) NGC 2681. (c) NGC 3814 . (d) NGC 4736. (e) NGC 4579. $(f)$ NGC 4736. $(g)$ NGC 5055. ( $h$ ) NGC 6946. (i) NGC 7331. ( $j$ ) M51 Note that the CO image does not cover M51B. $(k)$ M83. $(l)$ M101. 


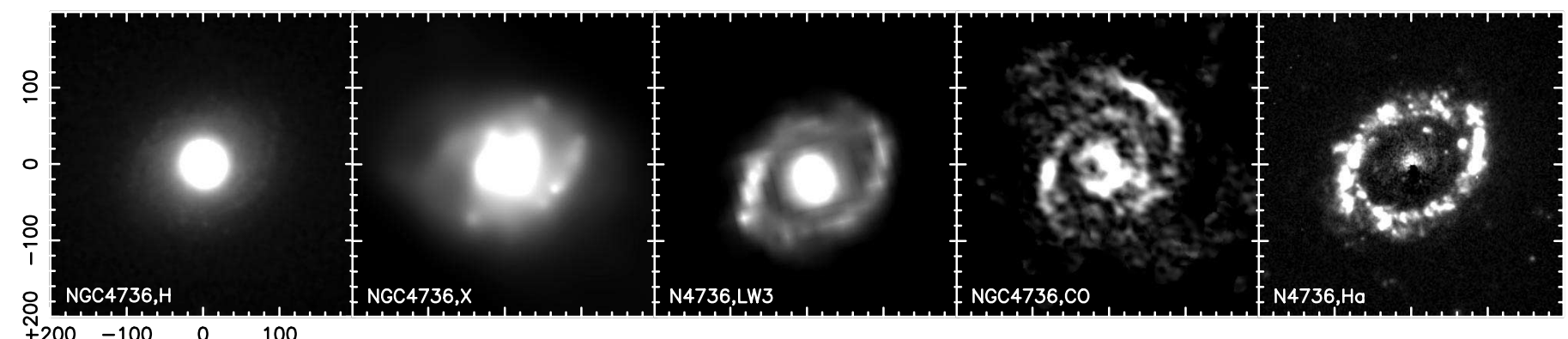

FIG. $1 f$

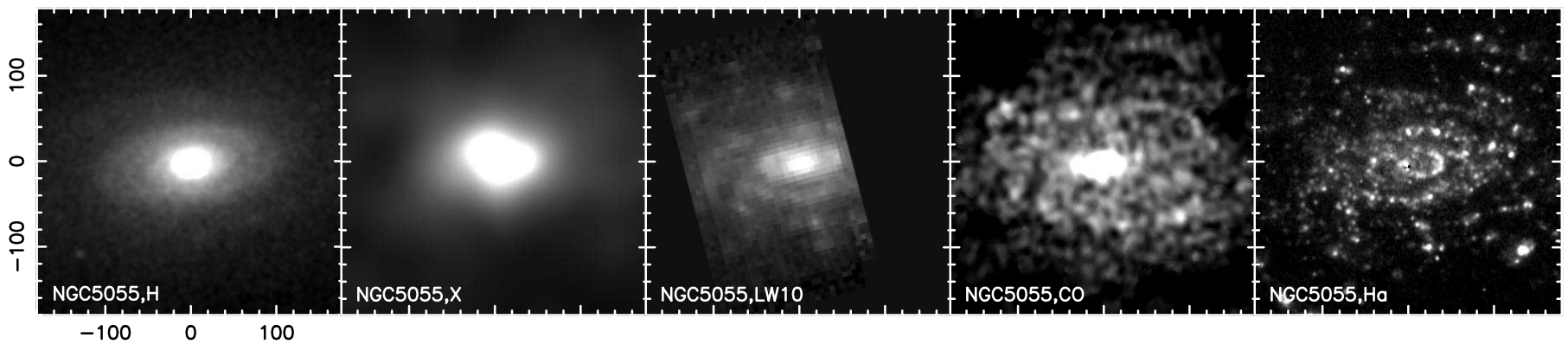

FIG. $1 g$

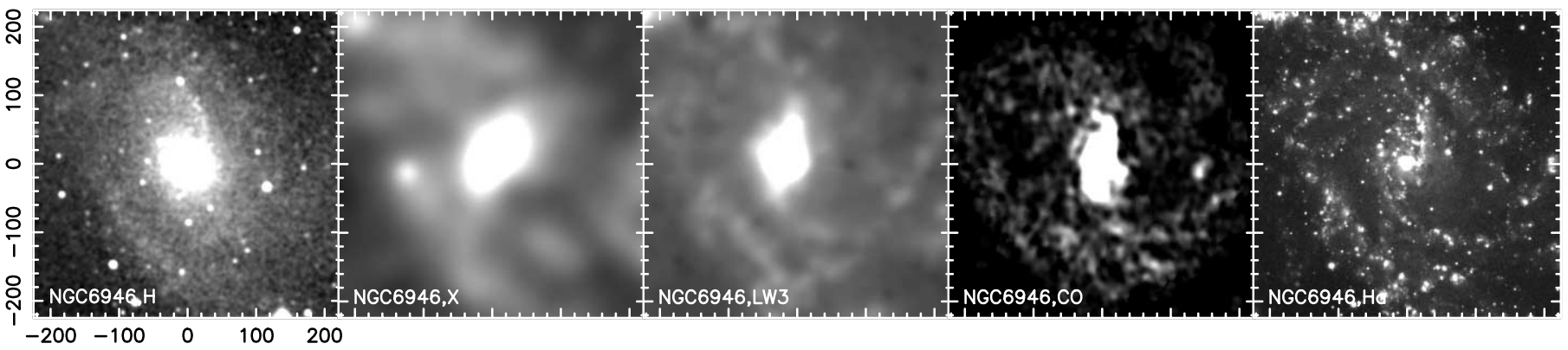

FIG. $1 h$

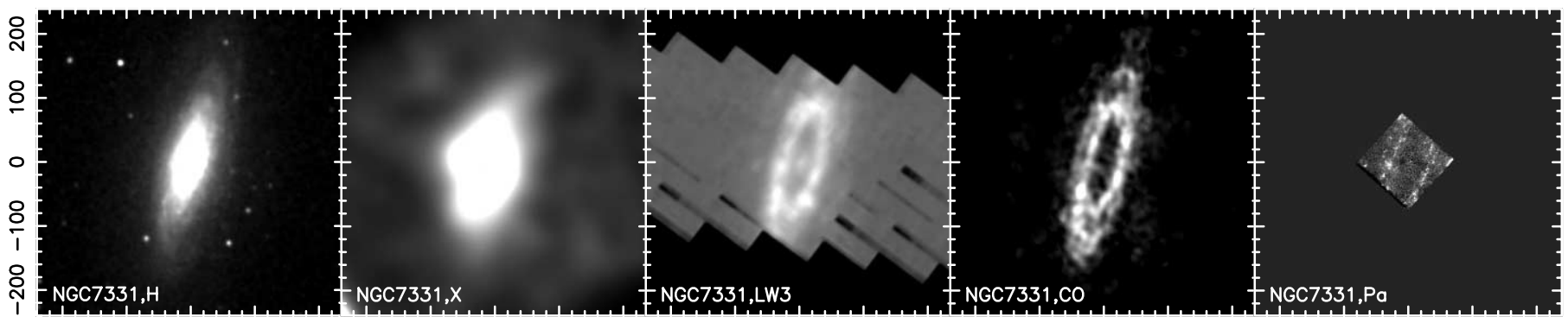

FIG. $1 i$

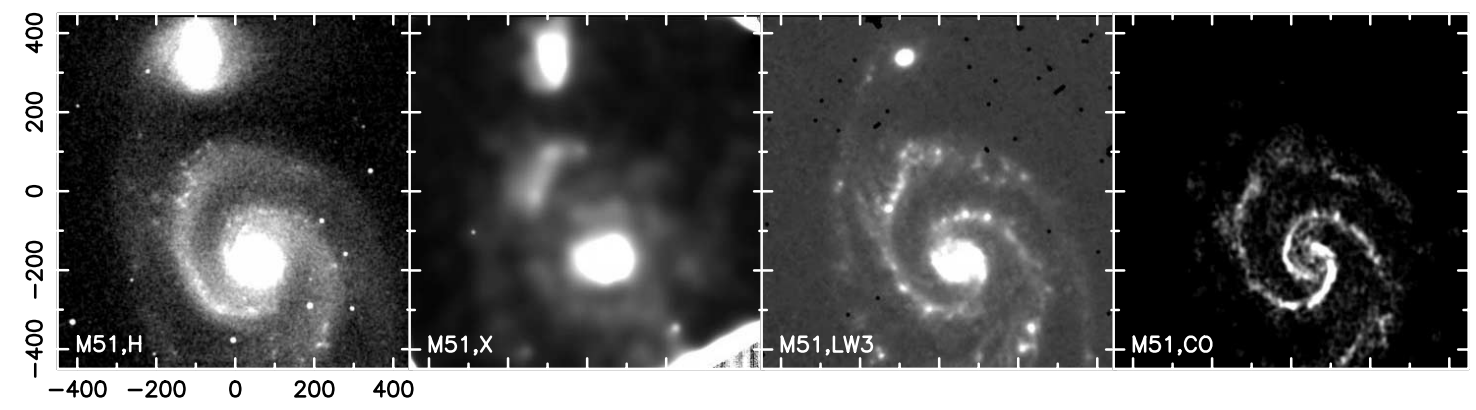

FIG. $1 j$ 

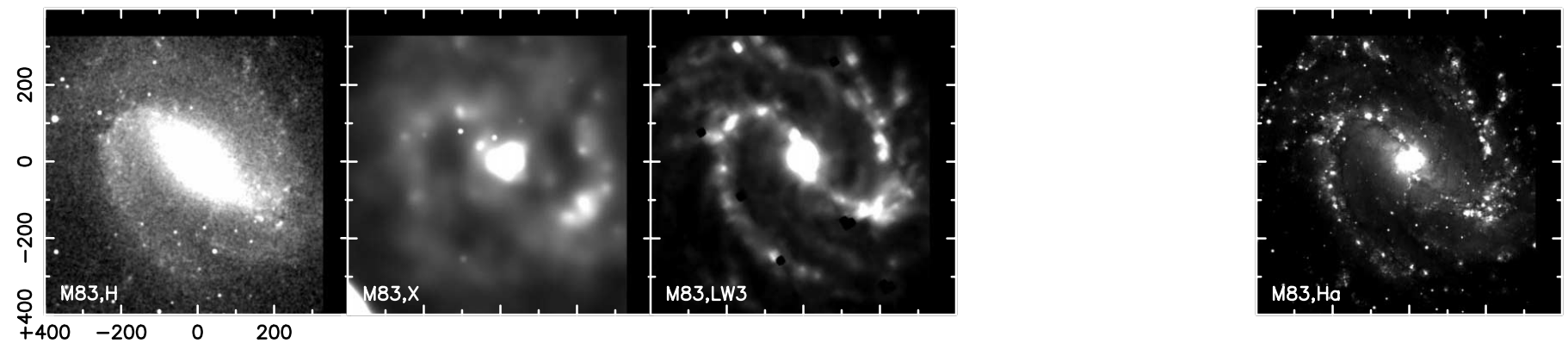

FIG. $1 k$

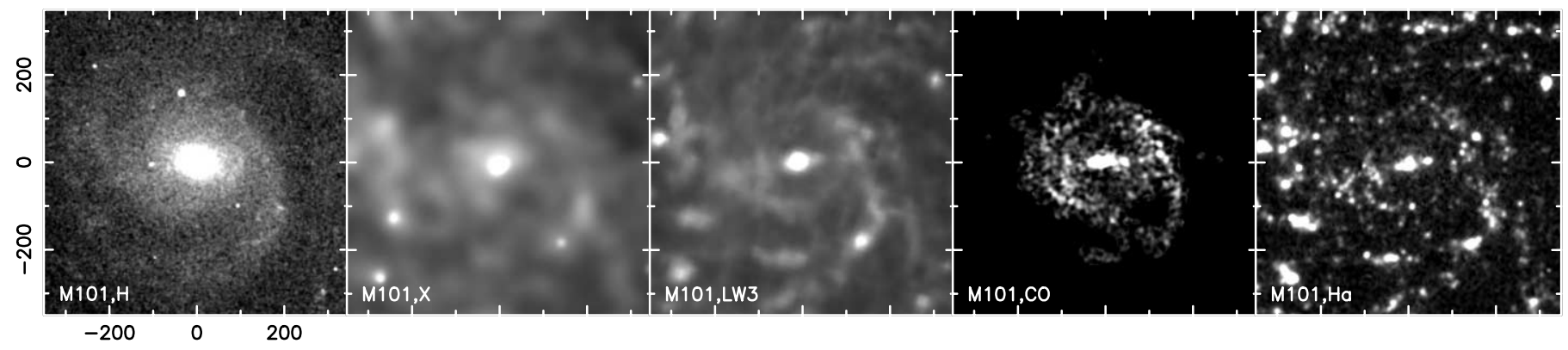

Fig. $1 l$

Space Observatory (ISO) showing emission from warm dust, which provides a tracer of recent star formation.

For NGC 2403, NGC 3184, NGC 6946, and M83 we use $\mathrm{H} \alpha$ images by Larsen \& Richtler (1999); for NGC 4579 and NGC 4303, those by Koopman et al. (2001); for NGC 4736, NGC 5055, and for M101, those by Wong \& Blitz (2002). Mid-infrared at 12 or $15 \mu \mathrm{m}$ images observed by the instrument ISOCAM are those made available on the NASA extragalactic database (NED) by Roussel et al. (2001) and Bendo et al. (2002). For NGC 7331 we downloaded the $15 \mu \mathrm{m}$ image discussed by Smith (1998) from the ISO archive. The comparison images are shown in Figure 1 with the X-ray images of the diffuse emission in each galaxy.

\section{RESULTS}

\subsection{The Morphology of the Diffuse X-Ray Emission}

In comparing the diffuse X-ray images with the images of other wavelengths, we see that the $\mathrm{H} \alpha$ and mid-infrared emission are coincident with each other and with the diffuse X-ray images (see M83 for example). The mid-infrared emission is composed of both continuum and polycyclic aromatic hydrocarbon warm dust components and scales with the rate of star formation (Roussel et al. 2001; Bendo et al. 2002), as would be expected by dust radiatively reprocessing the interstellar radiation field. The molecular gas (traced in $\mathrm{CO}$ ), from which the stars are formed, also tends to be coincident with the diffuse $\mathrm{X}$-ray emission, although it does not resemble the X-ray emission as closely as the $\mathrm{H} \alpha$ and mid-IR images. This is noticeable in the nucleus of M51, where the CO emission is not as prevalent as the X-ray or mid-infrared emission. Other discrepancies include the northwest side of NGC 4736 and the southern arm of NGC 3184, which contain prominent spiral arms in $\mathrm{CO}$ emission but little mid-infrared, X-ray, or $\mathrm{H} \alpha$ emission associated with them. There is also a region with a similar strong CO concentration to the south of M51's nucleus and little ongoing star formation (see Henry et al. 2003 for a discussion of the spiral arm asymmetries and efficiency of star formation in M51).

The association of diffuse X-ray morphology and that seen in $\mathrm{H} \alpha$ is similar to that found in previous studies (e.g., Strickland et al. 2004a; Fraternali et al. 2002). However, most previous studies have focused on individual $\mathrm{H}$ II regions or filaments associated with coronal emission and outflows in edge-on galaxies (e.g., Fraternali et al. 2002; Strickland et al. 2004a; Wang et al. 2001). Because we have focused on spiral galaxies that are not at high inclination (except for NGC 7331), we are better able to view the changes in morphology across the disks of the galaxies. We find a clear correspondence between the morphology of the diffuse X-ray emission and the specific locations of active sites of star formation.

Except for NGC 2403, which is irregular, all the galaxies in our sample emit diffuse X-rays from their centers. Galaxies NGC 3184, NGC 4303, NGC 4736, M51, M83, and M101 show spiral structure in the diffuse X-ray emission, coinciding with star formation in the spiral arms, as traced in either $\mathrm{H} \alpha$ or in the mid-infrared ISOCAM images. The emission from the galaxy centers is approximately radially symmetric, whereas that from the spiral arms is located in linear features lying along the arms. Because we see a difference in X-ray morphology between the spiral arms and the galaxy centers, we compare the two regions separately.

\subsection{The Diffuse X-Ray Emission in the Spiral Arms}

Most but not all of the spiral galaxies display X-ray emission along spiral arms. Galaxies NGC 3184, NGC 4303, NGC 4736, M51, M83, and M101 show prominent spiral structure in the diffuse X-ray emission, coinciding with star formation in the spiral arms, as traced by $\mathrm{H} \alpha$ or mid-infrared emission. NGC 4303, NGC 4736, and M101 show weaker spiral structure, whereas that in M51, M83, and NGC 3184 is particularly strong and open (not tightly wound). Little diffuse X-ray gas was detected in the outer regions of NGC 6946; however, NGC 6946 has a high Galactic absorption column, 


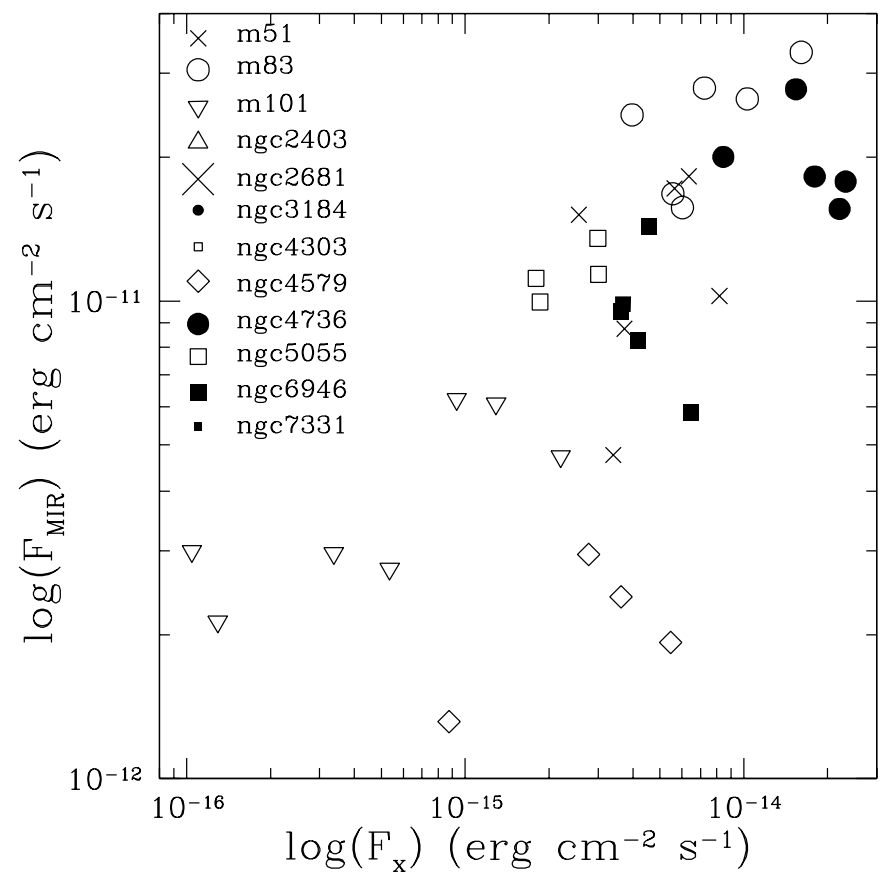

FIG. 2.-Comparison between mid-infrared and X-ray fluxes from $20^{\prime \prime}$ radii apertures in different regions along the spiral arms. The mid-infrared fluxes are measured from the 12 or $15 \mu \mathrm{m}$ ISOCAM images using $\nu F_{\nu}$. The X-ray fluxes are $0.1-2.0 \mathrm{keV}$ unabsorbed fluxes computed assuming a MEKAL (Liedahl et al. 1995; Mewe et al. 1985, 1986) spectrum with $k T=0.45 \mathrm{keV}$ and corrected for Galactic absorption. Positions in different galaxies are shown with different shaped data points. The mid-infrared fluxes are about 3-4 orders of magnitude above those seen in the X-rays. The points on the lower right are from NGC 4579, which contains an AGN (Eracleous et al. 2002). We observe a strong correlation between the diffuse X-ray and the mid-IR emission.

which may have affected our ability to detect the soft diffuse $\mathrm{X}$-ray emission.

Previous studies have shown that the soft X-ray luminosity of entire spiral galaxies is proportional to the total infrared luminosity (Read \& Ponman 2001; Strickland et al. 2004b), a direct indicator of the current star formation rate (Kennicutt 1998). Because we can observe the spatial distribution of both the diffuse X-ray emission and the current sites of star formation, we can compare the X-ray and mid-IR fluxes measured at different regions in each galaxy. Because longerwavelength infrared data lacks sufficient spatial resolution, we opt to use the mid-infrared ISO images. However, the total farinfrared bolometric flux used in previous studies can be estimated from the mid-infrared flux and has been empirically measured to be about 14 times the mid-infrared flux at $12 \mu \mathrm{m}$ (estimated as $\nu F_{\nu}$ ) for a wide range of galaxy luminosities (Spinoglio et al. 1995). We have opted to use the mid-infrared ISOCAM images as a tracer of recent star formation, rather than the $\mathrm{H} \alpha$ images because they were observed in a similar fashion with the same camera on the same telescope and thus are more uniform in their calibration than the available $\mathrm{H} \alpha$ images. The mid-IR images also have the advantage that they do not require a correction for extinction.

In Figure 2 we plot X-ray and mid-infrared fluxes measured in $20^{\prime \prime}$ radius apertures at different locations along the spiral arms. These locations were chosen in regions of high X-ray or mid-infrared surface brightness. We measured fluxes at different positions and angles, covering more than one quadrant in each galaxy. For those galaxies with lower surface bright- ness X-ray and mid-infrared emission, locations were chosen randomly in the spiral arms.

Good-quality spectra are difficult to extract from the outer parts of these galaxies because of the low count rates; consequently, we did not determine conversions between count rates and fluxes based on individual fitted emission models. We fitted spectra extracted from the brighter (central) components of a few galaxies and used the resulting model to convert between counts and flux for other regions. However, the spectra from the spiral arms are not necessarily the same as those from the nuclei of the galaxies, so a more accurate analysis would require spectral fitting in the spiral arms as well as in the nucleus. We note that Kuntz et al. (2003) measured little difference in the spectra extracted from high and low surface brightness regions in M101.

Background-subtracted spectra were extracted from the brighter galaxy centers of M51, M83, NGC 5055, NGC 4736, and NGC 7331 and corrected for time-dependent molecular absorption and changes in quantum efficiency with the XSPEC routine acisabs. To convert between counts $\mathrm{cm}^{-2} \mathrm{~s}^{-1}$ and unabsorbed 0.1-2.0 keV flux, we used an XSPEC MEKAL model with $k T=0.45 \mathrm{keV}$ and an abundance of 0.1 solar, which provided a reasonable fit to spectra extracted from the brighter central components of these galaxies. When Chandra spectra of diffuse emission in nearby galaxies are fitted with singlecomponent hot plasma models, they have been better fitted by those with extremely low abundances. This problem has been discussed by Strickland et al. (2000), Wang et al. (2001), and others. Here we do not attach any undue significance to the parameters of these fits but use them to derive conversion factors that we use to estimate physical quantities. We have noted that the conversion factors are insensitive to the value of the abundance used.

Background-subtracted fluxes were corrected for Galactic absorption based on Galactic H i column depth in the direction of each galaxy but not for absorption internal to each galaxy. The data points used in Figure 2 are listed in Table 2.

From Figure 2 we see that the X-ray flux in different regions along the spiral arms of these galaxies is correlated with that in the mid-infrared. We find that the correlation between X-ray emission and star formation previously seen on whole galaxy scales also exists on smaller scales along individual spiral arms, with the exception of NGC 4736 and NGC 6946, which do not show correlations in the X-ray/mid-infrared flux ratio in individual regions. However, these two galaxies still remain similar in their mid-infrared to X-ray flux ratio compared to other galaxies in the sample. NGC 4579 is the only galaxy with a significantly higher X-ray to infrared flux ratio than the other galaxies. This is probably due to its active galactic nucleus (AGN; discussed recently by Eracleous et al. 2002). Activity from the AGN has probably increased the quantity of diffuse X-ray emission at distances 3-7 kpc from the nucleus. Because of this large distance from the nucleus, we infer that the X-ray gas must have been heated mechanically rather than radiatively.

We support the conclusions of Young et al. (2001), who found that the starburst is not the dominant source of soft extended X-ray emission in Seyfert galaxies.

In the spiral arms of the other galaxies, the flux of X-ray emission is about 4 orders of magnitude lower than the midinfrared emission. Using the mid-infrared fluxes to estimate the total infrared bolometric fluxes, we estimate that the X-ray fluxes are 5 orders of magnitude below that, comprising the 
TABLE 2

X-Ray and Mid-IR Fluxes in the Spiral Arms

\begin{tabular}{|c|c|c|}
\hline Galaxy & $\begin{array}{c}\text { X-Ray } \\
\left(\text { ergs cm } \mathrm{cm}^{-2} \mathrm{~s}^{-1} \text { ) }\right.\end{array}$ & $\begin{array}{c}\text { Mid-IR } \\
\left(\text { ergs } \mathrm{cm}^{-2} \mathrm{~s}^{-1}\right)\end{array}$ \\
\hline \multirow[t]{6}{*}{ 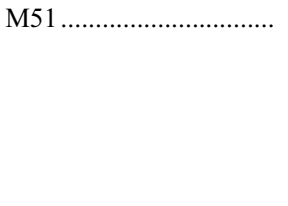 } & $6.4 \mathrm{E}-15$ & $1.8 \mathrm{E}-11$ \\
\hline & $8.2 \mathrm{E}-15$ & $1.0 \mathrm{E}-11$ \\
\hline & $5.6 \mathrm{E}-15$ & $1.7 \mathrm{E}-11$ \\
\hline & $3.7 \mathrm{E}-15$ & $8.7 \mathrm{E}-12$ \\
\hline & $3.4 \mathrm{E}-15$ & $4.8 \mathrm{E}-12$ \\
\hline & $2.6 \mathrm{E}-15$ & $1.5 \mathrm{E}-11$ \\
\hline \multirow[t]{6}{*}{ 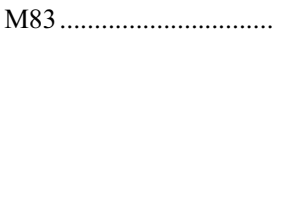 } & $1.6 \mathrm{E}-14$ & $3.3 \mathrm{E}-11$ \\
\hline & $1.0 \mathrm{E}-14$ & $2.7 \mathrm{E}-11$ \\
\hline & $6.0 \mathrm{E}-15$ & $1.6 \mathrm{E}-11$ \\
\hline & $4.0 \mathrm{E}-15$ & $2.5 \mathrm{E}-11$ \\
\hline & $7.2 \mathrm{E}-15$ & $2.8 \mathrm{E}-11$ \\
\hline & $5.6 \mathrm{E}-15$ & $1.7 \mathrm{E}-11$ \\
\hline \multirow[t]{7}{*}{ M101 ................................ } & $2.2 \mathrm{E}-15$ & $4.7 \mathrm{E}-12$ \\
\hline & $5.4 \mathrm{E}-16$ & $2.7 \mathrm{E}-12$ \\
\hline & $1.3 \mathrm{E}-15$ & $6.1 \mathrm{E}-12$ \\
\hline & $1.0 \mathrm{E}-16$ & $3.0 \mathrm{E}-12$ \\
\hline & $3.4 \mathrm{E}-16$ & $3.0 \mathrm{E}-12$ \\
\hline & $1.3 \mathrm{E}-16$ & $2.1 \mathrm{E}-12$ \\
\hline & $9.3 \mathrm{E}-16$ & $6.2 \mathrm{E}-12$ \\
\hline \multirow[t]{4}{*}{ 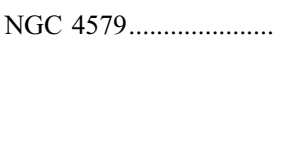 } & $5.5 \mathrm{E}-15$ & $1.9 \mathrm{E}-12$ \\
\hline & $2.8 \mathrm{E}-15$ & $2.9 \mathrm{E}-12$ \\
\hline & $3.6 \mathrm{E}-15$ & $2.4 \mathrm{E}-12$ \\
\hline & $8.8 \mathrm{E}-16$ & $1.3 \mathrm{E}-12$ \\
\hline \multirow{5}{*}{ 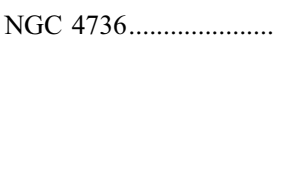 } & $2.3 \mathrm{E}-14$ & $1.8 \mathrm{E}-11$ \\
\hline & $1.8 \mathrm{E}-14$ & $1.8 \mathrm{E}-11$ \\
\hline & $2.2 \mathrm{E}-14$ & $1.6 \mathrm{E}-11$ \\
\hline & $1.5 \mathrm{E}-14$ & $2.8 \mathrm{E}-11$ \\
\hline & $8.5 \mathrm{E}-15$ & $2.0 \mathrm{E}-11$ \\
\hline \multirow[t]{4}{*}{ 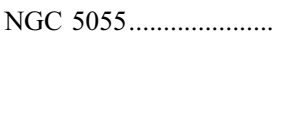 } & $1.8 \mathrm{E}-15$ & $1.1 \mathrm{E}-11$ \\
\hline & $1.9 \mathrm{E}-15$ & $10.0 \mathrm{E}-12$ \\
\hline & $3.0 \mathrm{E}-15$ & $1.4 \mathrm{E}-11$ \\
\hline & $3.0 \mathrm{E}-15$ & $1.1 \mathrm{E}-11$ \\
\hline \multirow[t]{5}{*}{ 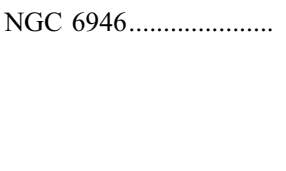 } & $3.7 \mathrm{E}-15$ & $9.9 \mathrm{E}-12$ \\
\hline & $4.2 \mathrm{E}-15$ & $8.3 \mathrm{E}-12$ \\
\hline & $6.5 \mathrm{E}-15$ & $5.9 \mathrm{E}-12$ \\
\hline & $4.6 \mathrm{E}-15$ & $1.4 \mathrm{E}-11$ \\
\hline & $3.6 \mathrm{E}-15$ & $9.5 \mathrm{E}-12$ \\
\hline
\end{tabular}

Notes.-Fluxes are measured in $20^{\prime \prime}$ apertures in the spiral arms of given galaxies. X-ray fluxes have been corrected for Galactic absorption. Both X-ray and mid-infrared fluxes have been background-subtracted. Mid-infrared fluxes have been measured from ISOCAM 12 or $15 \mu \mathrm{m}$ images using $\nu F_{\nu}$ and calibration factors given by the $I S O$ archive. A plot of these data is shown in Fig. 2.

bolometric far-infrared fluxes. This is 1 order of magnitude below the correlation found by Read \& Ponman (2001) and Strickland et al. (2004b). Since the galaxies in our sample contain both starbursts (M83) and dwarf galaxies (NGC 2403), it covers a range of luminosities similar to the samples considered by Read \& Ponman (2001) and Strickland et al. (2004b). The discrepancy between our estimated X-ray to infrared ratio and previous work could be due to the temperature we assumed to convert between counts and flux.

\subsection{Nuclear Diffuse X-Ray Emission}

Except for NGC 2403, which is irregular, all the galaxies in our sample emit diffuse X-rays from their centers. With the exception of NGC 7331, the nuclei of these galaxies are also sites of star formation. NGC 7331 has a star-forming region ring at about $8 \mathrm{kpc}$ from its nucleus and little star formation within this ring. However, the X-ray emission peaks at the galaxy nucleus and not at the location of the molecular ring. In NGC 7331, the X-ray emission could be associated with the bulge of the galaxy, the star-forming ring or its faint Seyfert 2 nucleus. The bulge in the X-ray emission map northeast of the nucleus that resembles cones seen in other edge-on galaxies (e.g., Strickland et al. 2004a) suggests that some of the X-rayemitting gas was heated in the galaxy center, by the AGN or a past episode of star formation.

We can consider three possible dominant energy sources resulting in the presence of hot X-ray-emitting gas in the centers of these galaxies: stellar winds and supernovae associated with an old bulge population, superwinds driven by active star formation, and an active galactic nucleus. Since the $\mathrm{X}$-ray emission is not closely associated with individual spiral arms in the centers of these galaxies, and the morphologies are nearly radially symmetric, a galactic fountain type scenario is unlikely. To discriminate between the possible energy sources, in Figure 3 we plot diffuse X-ray fluxes compared to those in the same apertures at $H$ band (based on the 2MASS images), in CO (based on the BIMA-SONG data) and at 12 or $15 \mu \mathrm{m}$ (based on the ISOCAM images). We have chosen to plot fluxes rather than luminosities to minimize the chance of seeing a false correlation caused by the different galaxy distances. Apertures centered on the galaxy nucleus were adjusted to include the entire central diffuse $\mathrm{X}$-ray component and have radii that range from $10^{\prime \prime}$ to $25^{\prime \prime}$. The $0.1-2.0 \mathrm{keV}$ unabsorbed X-ray fluxes have been measured in the same way as those along the spiral arms (see §3.2) and are listed in Table 3. For the irregular galaxy NGC 2403, we centered the aperture on the brightest region of the galaxy as seen in the 2MASS $1.6 \mu$ m image.

If stellar winds and supernovae associated with older stars were responsible for the X-ray emission in the nuclear regions of these galaxies, then we would expect a correlation between the nuclear X-ray flux and that at $1.6 \mu \mathrm{m}$ in $H$ band, tracing the old stellar population. To test this possibility we show in Figure 3a a comparison between the nuclear X-ray fluxes and the $H$-band magnitude measured from the 2MASS images in the same regions. The X-ray fluxes range over 4 orders of magnitude, while the $H$-band fluxes range over 2 orders of magnitude. The range of these numbers is not strongly affected by the scatter in distances, since the nearest galaxy in our sample is only 3 times nearer than the farthest one. We find a correlation between the two fluxes, suggesting that an old stellar population may be associated with the diffuse X-ray emission. Either winds and supernovae from an old stellar population contribute to the X-ray-emitting gas or the mass of the bulge provides a gravitational potential well that prevents the X-ray gas from escaping the galaxy.

ROSAT studies of early-type galaxies confirmed the correlation between optical and X-ray luminosity previously revealed by Einstein studies (Brown \& Bregman 1998), finding that the X-ray luminosity was about $4-5$ orders of magnitude fainter than the optical luminosity. The points in Figure $3 a$ are consistent with this, suggesting that the diffuse emission in bulges could be an extension of that seen in early-type galaxies.

Figure $3 b$ shows the X-ray and mid-infrared fluxes from the galaxy cores in the same apertures as for Figure $3 a$. We see a larger scatter in the X-ray and mid-infrared fluxes in the cores than in the spiral arms. Most of the galaxies are consistent with the average mid-infrared to X-ray flux ratio seen in the spiral arms (see Fig. 2); however, there are some outlying points. Again, NGC 4579 has a higher X-ray to emitted infrared flux ratio that is very likely due to the proximity of the AGN. NGC 6946 has an X-ray/mid-infrared flux ratio of 


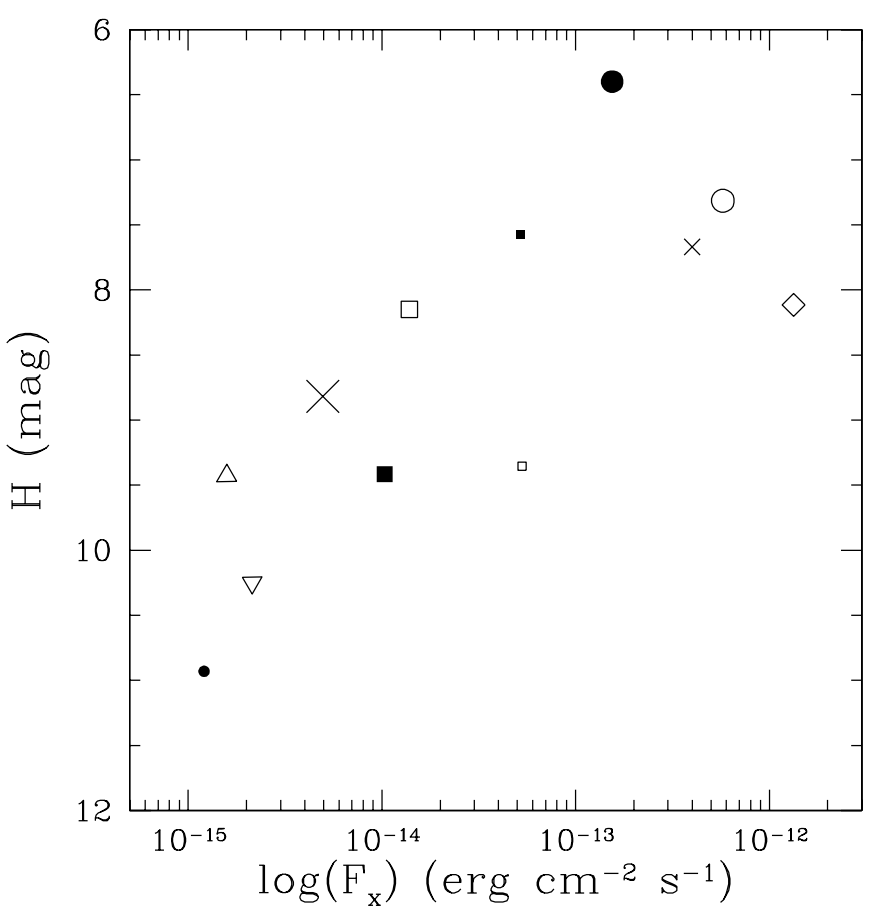

FIG. $3 a$

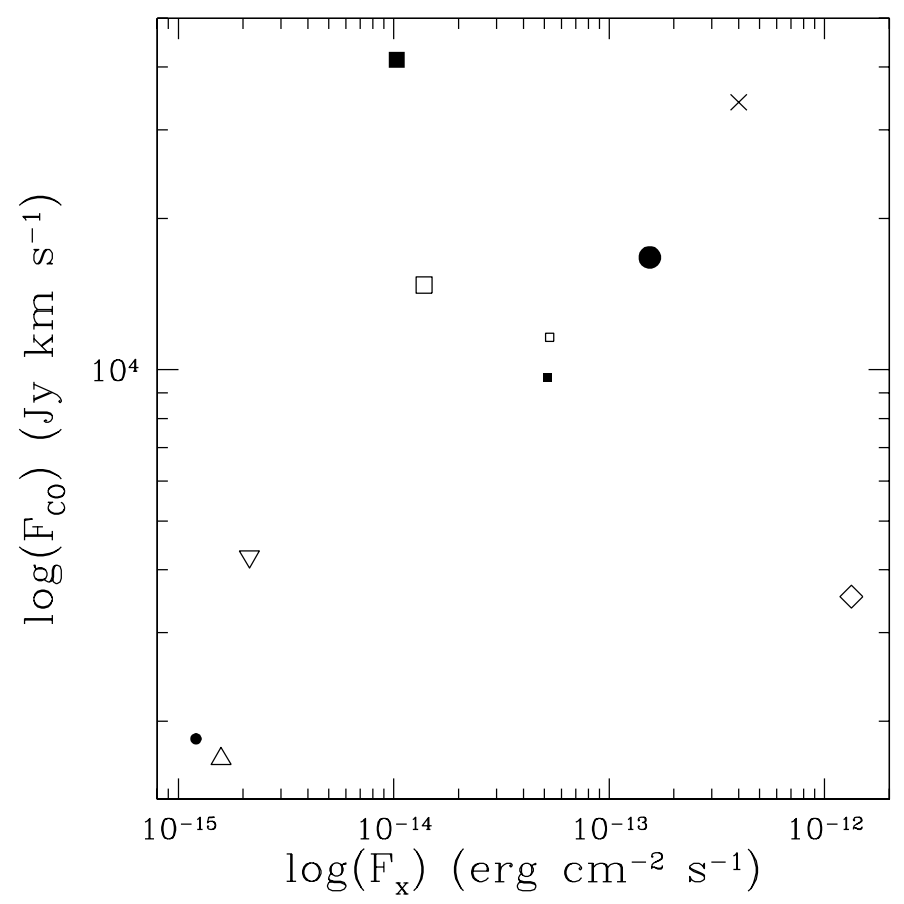

FIG. $3 c$

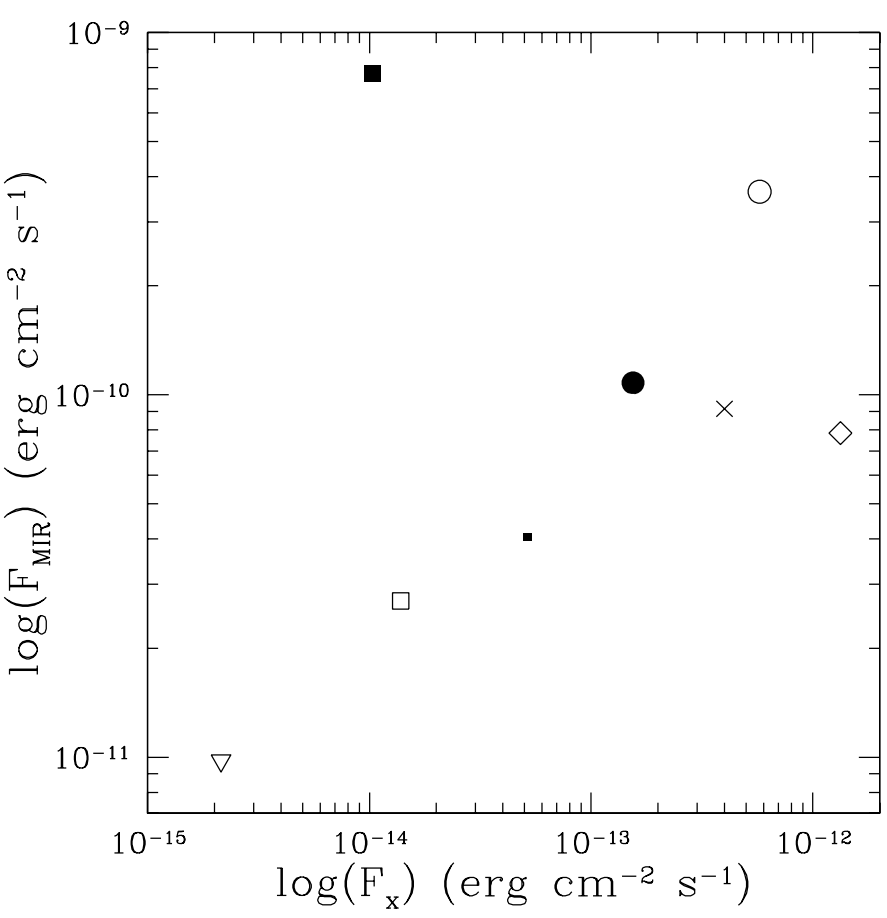

FIG. $3 b$

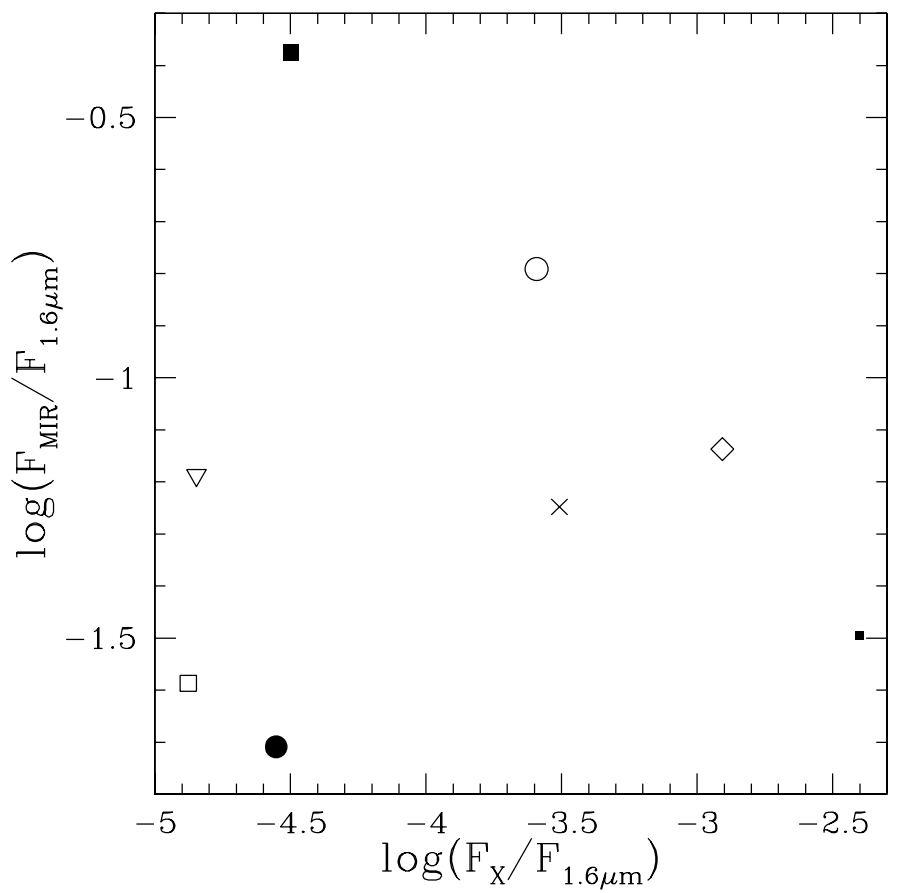

Fig. $3 d$

Fig. 3. - Comparison between central diffuse X-ray fluxes and those at other wavelengths. The data point shapes are the same as shown in Fig. 2. Apertures were selected to include the entire nuclear diffuse X-ray emission component and range between $10^{\prime \prime}$ and $25^{\prime \prime}$ (fluxes and apertures are listed in Table 3). (a) We compare $\mathrm{X}$-ray diffuse emission fluxes ( $x$-axis) to the $H$-band magnitudes ( $y$-axis) as measured from the 2MASS images. We see a weak correlation between the bulge and nuclear diffuse X-ray fluxes. (b) A comparison between mid-infrared from ISOCAM images (tracing warm dust) and diffuse X-ray emission fluxes in the cores of the galaxies. There is more scatter in the X-ray to mid-IR core flux ratio than in the spiral arms. The outlying point in the upper left is NGC 6946, which may have a reduced measured X-ray flux because of high Galactic extinction. The one at the far lower right is NGC 4579, which contains an AGN. (c) A comparison between CO (from BIMA-SONG data) and X-ray fluxes in the centers of the galaxies. Again, the outlying points are NGC 6946 (upper left) and NGC 4579 (lower right). (d) Ratio of mid-IR and $1.6 \mu \mathrm{m}$ flux vs. ratio of X-ray and $1.6 \mu \mathrm{m}$ flux. There is little correlation seen in this plot, suggesting that more than one factor determines the quantity of diffuse X-ray emission in the centers of galaxies. 
TABLE 3

X-Ray, Mid-IR, CO, and 2MASS IR Core Measurements

\begin{tabular}{|c|c|c|c|c|c|c|}
\hline Galaxy & $\begin{array}{c}\text { X-Ray } \\
\left(\text { ergs } \mathrm{cm}^{-2} \mathrm{~s}^{-1}\right)\end{array}$ & $\begin{array}{c}\text { Mid-IR } \\
\left(\text { ergs } \mathrm{cm}^{-2} \mathrm{~s}^{-1}\right)\end{array}$ & $\begin{array}{c}\mathrm{CO} \\
\left(\mathrm{Jy} \mathrm{km} \mathrm{s} \mathrm{s}^{-1}\right)\end{array}$ & $\begin{array}{l}H \text { band } \\
\text { (mag) }\end{array}$ & $\begin{array}{l}\text { Aperture } \\
(\operatorname{arcsec})\end{array}$ & $\begin{array}{c}\text { Galactic } N_{\mathrm{H}} \text { Column } \\
\left(10^{20} \mathrm{~cm}^{-2}\right)\end{array}$ \\
\hline M51 ................... & $4.0 \mathrm{E}-13$ & $9.1 \mathrm{E}-11$ & $3.4 \mathrm{E} 4$ & 7.7 & 25 & 1.52 \\
\hline M83 .................... & $5.8 \mathrm{E}-13$ & $3.6 \mathrm{E}-10$ & $\ldots$ & 7.3 & 20 & 3.70 \\
\hline M101 .................. & $2.1 \mathrm{E}-15$ & $9.8 \mathrm{E}-12$ & $4.2 \mathrm{E} 3$ & 10.2 & 10 & 1.15 \\
\hline NGC $2403 \ldots \ldots \ldots . .$. & $1.6 \mathrm{E}-15$ & $\ldots$ & $1.7 \mathrm{E} 3$ & 9.4 & 20 & 4.17 \\
\hline NGC $2681 \ldots \ldots \ldots . . .$. & $4.9 \mathrm{E}-15$ & $\ldots$ & $\ldots$ & 8.8 & 10 & 2.48 \\
\hline NGC $3184 \ldots \ldots \ldots . .$. & $1.2 \mathrm{E}-15$ & $\ldots$ & $1.8 \mathrm{E} 3$ & 10.9 & 10 & 1.15 \\
\hline NGC 4303 .......... & $5.3 \mathrm{E}-14$ & $\ldots$ & $1.2 \mathrm{E} 4$ & 9.4 & 10 & 1.65 \\
\hline NGC $4579 \ldots \ldots \ldots$ & $1.3 \mathrm{E}-12$ & $7.8 \mathrm{E}-11$ & $3.5 \mathrm{E} 3$ & 8.1 & 20 & 2.51 \\
\hline NGC $4736 \ldots \ldots \ldots . .$. & $1.5 \mathrm{E}-13$ & $1.1 \mathrm{E}-10$ & $1.7 \mathrm{E} 4$ & 6.4 & 20 & 1.44 \\
\hline NGC $5055 \ldots \ldots \ldots . .$. & $1.4 \mathrm{E}-14$ & $2.7 \mathrm{E}-11$ & $1.5 \mathrm{E} 4$ & 8.1 & 15 & 1.30 \\
\hline NGC $6946 \ldots \ldots \ldots . .$. & $1.0 \mathrm{E}-14$ & $7.7 \mathrm{E}-10$ & $4.1 \mathrm{E} 4$ & 9.4 & 10 & 20.05 \\
\hline NGC $7331 \ldots \ldots \ldots . .$. & $5.2 \mathrm{E}-14$ & $4.1 \mathrm{E}-11$ & 9.7E3 & 7.6 & 20 & 8.25 \\
\hline
\end{tabular}

NoTES.-Values in the cores of galaxies for the given apertures for the diffuse X-ray, Mid-IR, CO, and 2MASS $H$-band images. X-ray fluxes have been corrected for the indicated Galactic absorption. X-ray, mid-IR, CO, and 2MASS IR have all been background-subtracted. These data points are plotted in Fig. 3.

$10^{-5}, 2$ orders of magnitude below the mean seen in the spiral arms. NGC 6946's low level of diffuse central X-ray emission could be due to its small bulge or because it had been quiescent until recently, as prior star-forming activity could affect the total quantity of diffuse nuclear X-ray emission. We note that the Galactic H I column toward NGC 6946 is high. If the diffuse emission from this galaxy is very soft, then we may have underestimated the flux from this component.

Since we see that the diffuse X-ray flux correlates with the infrared flux (and thus the star formation rate), it should also be correlated with the quantity of molecular gas. Figure $3 c$ shows X-ray and $\mathrm{CO}$ fluxes for the galaxy cores, using the same apertures. The $\mathrm{CO}$ fluxes were calculated using $\mathrm{CO}$ images from the BIMA-SONG survey and are also listed in Table 3. The fluxes are similarly scattered as in Figure $3 b$, forming a weak correlation with two outlying points, again corresponding to NGC 6946 (upper left) and NGC 4579 (lower right). Two of the galaxies in our sample were not included in the BIMA-SONG survey and, as such, are not included in this graph: M83 and NGC 2681.

To test the dependence of diffuse X-ray emission on supernovae from older stars and starburst activity, we plotted the ratio of mid-infrared to $1.6 \mu \mathrm{m}$ flux versus the ratio of $\mathrm{X}$-ray to $1.6 \mu \mathrm{m}$ flux (Fig. $3 d$ ). The lack correlation shown in the plot suggests that current star formation is not the only factor affecting the quantity of diffuse X-ray flux. Unfortunately, many of the high points in Figure $3 d$ are also high in Figures $3 a$ and $3 b$, implying that the galaxies with brighter $\mathrm{X}$-ray nuclear components have bigger bulges and also higher levels of active star formation near their centers. This makes it difficult to discriminate between scenarios for producing the X-ray gas. We checked the nuclear spectral classification (listed in Table 1) of the galaxies in this plot and found no obvious correspondence between nuclear type and X-ray/ infrared color.

In short, we find weak correlations between central diffuse $\mathrm{X}$-ray flux and bulge brightness and between the X-ray flux and the current rate of star formation. The correlation between the X-ray flux and mid-IR flux is weaker than that seen in the spiral arms, suggesting that other factors are affecting the $\mathrm{X}$-ray luminosity. These other factors could include the depth of the bulge potential, winds and supernovae from bulge stars, AGN activity, and previous episodes of star formation.

\subsection{Constraints on the Volume Filling Factor, Electron \\ Density, and Cooling Time of the X-Ray-emitting Gas}

As we mentioned above, we used the Sherpa analysis program to fit single-component hot plasma MEKAL models to spectra extracted from the brighter galaxy nuclei.

The MEKAL normalization, $K=10^{-14} /\left(4 \pi D_{\mathrm{cm}}^{2}\right) \int n_{e} n_{\mathrm{H}} d V$, required to fit these spectra, can be used to place constraints on the emission integral of the X-ray-emitting gas and consequently on its volume filling factor, $f_{v}$, and electron density, $n_{e}$. Here $D_{\mathrm{cm}}$ is the angular size distance to the source in centimeters. The electron density can be estimated from the normalization factor

$$
n_{e}=0.13 \mathrm{~cm}^{-3} f_{v}^{-1 / 2}\left(\frac{h}{\mathrm{kpc}}\right)^{-1 / 2}\left(\frac{K}{10^{-4} A}\right)^{1 / 2}
$$

where $A$ is the aperture area in square arcseconds where we measured the count rate, and we have assumed that the emission integral $\mathrm{EI}=\int n_{e} n_{\mathrm{H}} d V \sim n_{e}^{2} f_{v} h a$, where $a$ is the area in $\mathrm{cm}^{2}$ where the count rate was measured. We have scaled the above expressions assuming a typical scale height $h \sim 1 \mathrm{kpc}$ corresponding to $20^{\prime \prime}$ for a nearby galaxy at a distance of $10 \mathrm{Mpc}$. This size scale would be appropriate for the central components of our galaxies if the central X-ray emission filled a spherical volume.

Estimated electron densities in the nuclear regions range from $n_{e} \sim 0.016 \mathrm{~cm}^{-3} f_{v}^{-1 / 2}(h / 1 \mathrm{kpc})^{-1 / 2}$ in the center of M101 to $n_{e} \sim 0.11 \mathrm{~cm}^{-3}$ (same scaling) in the center of M83. The irregular galaxy NGC 2403 has a somewhat lower $n_{e} \sim 0.012 \mathrm{~cm}^{-3}$ and NGC 4579, which contains a bright AGN, is higher with $n_{e} \sim 0.19 \mathrm{~cm}^{-3}$.

Given an estimated electron density and our assumed temperature, we can estimate a radiative cooling time for the hot gas, $t_{\text {cool }} \sim 10^{7} n_{e}^{-1} \mathrm{yr}$, where $n_{e}$ is in $\mathrm{cm}^{-3}$. This results in a cooling time of $t_{\text {cool }} \sim 4.5 \times 10^{7} f_{v}^{1 / 2}(h / 1 \mathrm{kpc})^{1 / 2} \mathrm{yr}$ for the gas near the center of NGC 4579 and $t_{\text {cool }} \sim 7 \times 10^{8}$ yr for NGC 2403 and M101. The other galaxy centers fall between these estimates. Except in the case of M83, there is no evidence for recent elevated rates of star formation that could have taken place within these estimated cooling timescales. It is unlikely that we are observing all of these nearby galaxies at a particular time following a recent burst of star formation that 
could have produced this X-ray gas. If the amount of X-ray gas in these galaxy centers is typical of normal galaxies, then the cooling times we have estimated are quite short. They would be even shorter if the filling factor or scale heights were lower. This suggests that the X-ray emission from the brighter galaxy centers has a fairly high volume filling factor and a large scale height, not inconsistent with previous studies of edge-on galaxies (Strickland et al. 2004a). We note that this problem with the cooling time has been previously discussed (Strickland et al. 2004a) and is somewhat less restrictive (by a factor of 3 or so) if the abundance of the hot gas is taken into account. This is because the emission integral depends on the number of free electrons and thus is approximately inversely proportional to metallicity. Our estimated densities could be a factor of a few too high and thus our estimated cooling time too high by the same factor.

Following the same procedures as we previously used for the nuclear regions, we can estimate the electron density and cooling time for the diffuse X-ray emission in the spiral arms. The count rates in the arms using $20^{\prime \prime}$ apertures average around $10^{-6}$ counts $\mathrm{cm}^{-2} \mathrm{~s}^{-1}$, corresponding to an average electron density $n_{e} \sim 0.006 \mathrm{~cm}^{-3} f_{v}^{-1 / 2}(h / 1 \mathrm{kpc})^{-1 / 2}$, and an associated cooling time of $t_{\text {cool }} \sim 1.4 \times 10^{9} \mathrm{yr} f_{v}^{1 / 2}(h / 1 \mathrm{kpc})^{1 / 2}$.

The electron densities in the spiral arms are naturally lower than those in the galaxy centers. However, the diffuse X-ray emission in the outer parts of the galaxy is mostly located in the spiral arms and not in between the arms. For the hot gas to be confined to regions near the spiral arms, we require that hot gas produced in the arms cool off before it rotates away from the spiral arms. Second, less hot gas can be produced interarm.

Hot gas produced in the arms can cool radiatively or by adiabatic expansion. In either case, the cooling time must not be longer than the time it takes for the spiral pattern to travel a large angle through the galaxy. The galaxies considered here range in rotational velocities estimated from their $\mathrm{H}$ I line width and apparent inclinations, from $150 \mathrm{~km} \mathrm{~s}^{-1}$ (for the dwarf NGC 2403) to $300 \mathrm{~km} \mathrm{~s}^{-1}$ (NGC 7331). For a galaxy rotating at $200 \mathrm{~km} \mathrm{~s}^{-1}$, the rotational period is $0.3 \mathrm{Gyr}$ at a radius of $10 \mathrm{kpc}$ from the galaxy nucleus. Spiral patterns travel at an angular rotation rate that is approximately the same size as the rotational velocity divided by the radius. The radial extent of the open spiral arms implies that entire arms cannot be near their corotation resonance. For the X-ray emission to appear in the spiral arms and be absent between them, the hot gas produced in the arms must cool faster than the rotational period. Since our estimated radiative cooling time was much larger than the angular rotation rate for $f_{v} h \sim 1 \mathrm{kpc}$, we infer that the product of the scale height and volume filling factor is probably lower than this value.

We now consider cooling by adiabatic expansion. If the gas expands by a factor of 3 , then the intensity of the X-ray emission can drop by an order of magnitude and thus become undetectable interarm. The time for expansion depends on the sound speed $\sim 300 \mathrm{~km} \mathrm{~s}^{-1}, t_{\exp } \sim\left[\left(3 f_{v} h\right) / 300 \mathrm{~km} \mathrm{~s}^{-1}\right] \sim$ $10 \mathrm{Myr} f_{v} h_{\mathrm{kpc}}$. A time period of $10 \mathrm{Myr}$ would cause the hot gas to lag the spiral arms. This suggests that even if cooling took place through adiabatic expansion, to keep the detected hot gas confined to the spiral arms, we would require a low volume filling factor and scale height $f_{v} h \lesssim 1 \mathrm{kpc}$.

Observations of edge-on galaxies have shown that the scale height of X-ray-emitting gas is seldom above a kpc (Strickland et al. 2004a) and is filamentary, consistent with our constraints based on the cooling time. The low scale height and volume filling factor is not inconsistent with the narrow appearance of the spiral arms and implies that the hot gas is not in hydrostatic equilibrium. Because the ratio of the sound speed in $10^{6} \mathrm{~K}$ gas to the rotational velocity is nearly 1 , gas in hydrostatic equilibrium would necessarily have $h / r \sim 1$. If the volume filling factor is indeed lower than 1 , then the density in the X-ray-emitting medium could be high enough $n_{e} \sim 1 \mathrm{~cm}^{-3}$ to correspond to what we might expect from $\mathrm{X}$-ray gas originating from supernovae in dense environments. To be consistent with such an electron density, we would infer that $f_{v} \lesssim 0.01$.

In summary, the high emission measures in the centers of these galaxies suggest that the volume filling factor and scale heights of the X-ray-emitting gas are high $(h \sim$ a few kiloparsecs), whereas those in the spiral arms suggest the opposite, that the volume filling factor and scale heights are low ( $h$ less than a few $\mathrm{kpc}$ ). It is tempting to consider a picture in which a galactic fountain type scenario operates in the outer parts of these galaxies and superwinds are driven from galaxy centers.

\subsection{Comparison of Diffuse X-Ray Emission and Star-forming Regions with the Predictions of Population Synthesis Models}

We now consider the predictions of population synthesis models. Density variations associated with spiral density waves are expected to be large in molecular gas (because of the interstellar medium [ISM] shocks) and products associated with star formation ( $\mathrm{H} \alpha$ and mid-infrared emission) but weaker in the old stellar component seen in the near-infrared (e.g., Gonzalez \& Graham 1996). In spiral galaxies, the star formation rate is highest just after a spiral arm passes through the ISM. $\mathrm{H} \alpha$ and mid-infrared emission peak when the massive young stars are formed. Population synthesis models (e.g., Leitherer \& Heckman 1995; Cervino et al. 2002) of instantaneous bursts predict a peak in $\mathrm{H} \alpha$ that lasts $\sim 3 \times$ $10^{6} \mathrm{yr}$ until the most massive stars leave the main sequence. The energy deposition rate from subsequent supernovae rises at this time and remains fairly flat until $\sim 3 \times 10^{7} \mathrm{yr}$ after the onset of the burst. This gives a characteristic timescale for the expected production of X-ray gas. Spiral arms contain H II regions as well as star clusters so clearly that there is a range of ages along the arm (or widths if we think of the spiral arm as a pattern passing through the disk of the galaxy). So we cannot think of the arm as a wave where at each point we see one and only one age after the burst. However, because $\mathrm{H}$ II regions can only last a few million years and the mechanical energy deposition from supernovae takes place over a timescale 10 times as long, we do expect a difference between the morphology seen in $\mathrm{H} \alpha$ or mid-IR compared to that seen in $\mathrm{X}$-ray. In particular, we expect that the X-ray emission will lag or be offset from the $\mathrm{H} \alpha$ and mid-infrared emission in the spiral arm.

The angular size of the lag depends on the timescale for supernovae to occur and the angular rotation rate of the disk compared to the spiral pattern. We expect larger angular offsets or lags in the center of the galaxy where the rotation periods are fast compared to the outer parts. At a distance of $5 \mathrm{kpc}$ from the nucleus and with a typical circular velocity of $200 \mathrm{~km} \mathrm{~s}^{-1}$, the rotational period of a star around the galaxy is $150 \mathrm{Myr}$. The timescale for supernovae to inject energy is about 30 million yr. This is one-fifth of the rotational period and thus would correspond to an angular width of $70^{\circ}$. For radii twice this, the corresponding angular width would be $45^{\circ}$, large enough to be easily detectable in our figures that compare the X-ray emission to that seen in $\mathrm{H} \alpha$ or the mid-IR. 

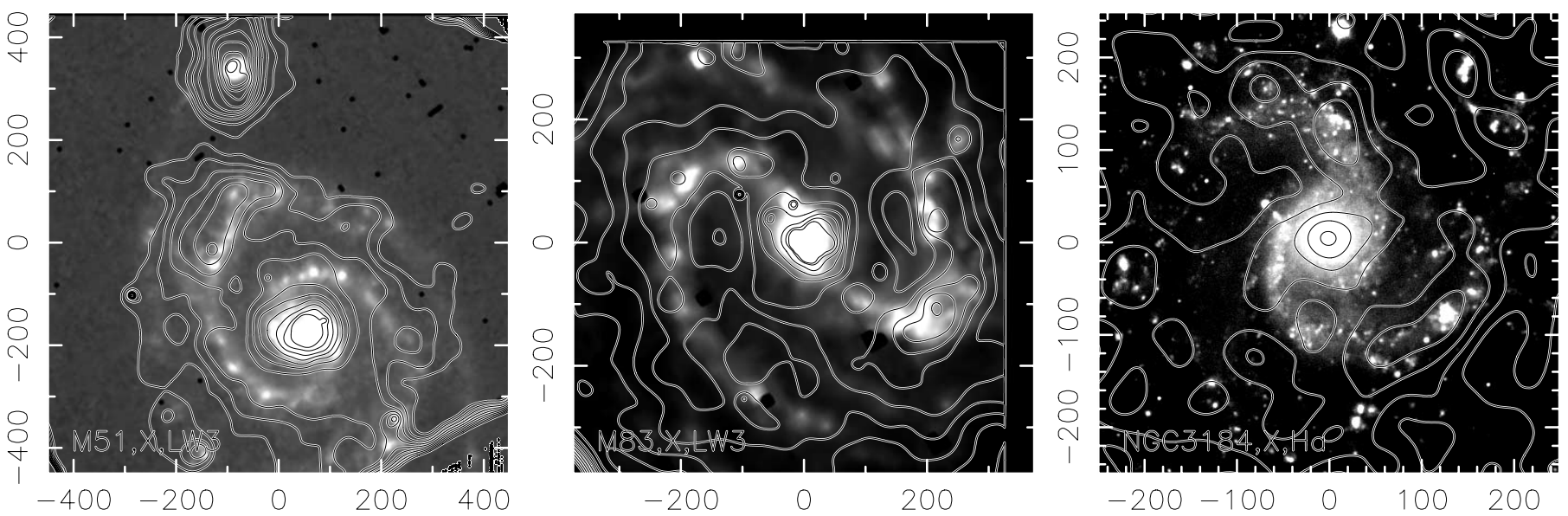

FIG. 4.-Diffuse X-ray contours on top of mid-infrared $15 \mu \mathrm{m}$ emission ( gray scale) for M51, M83, and H $\alpha$ (gray scale) for NGC 3184. In the outer spiral arms of M51 and M83, some of the X-ray emission extends to larger radii than the sites of star formation as seen in the mid-infrared image. There is little evidence for this in NGC 3184, where the X-ray emission lies centered on the regions of active star formation. Population synthesis models predict a constant energy deposition rate due to supernovae over a time period that is 10 times longer than the lifetime of the massive stars associated with the $\mathrm{H}$ II regions, and so we would have expected that the X-ray emission would significantly lag the sites of active star formation. The narrowness of the spiral arms seen in the diffuse component and close association with the site of active star formation suggest that interarm supernovae produce less soft X-ray emission than those near the site of massive star formation.

The narrowness of the arms seen in the $\mathrm{H} \alpha$ and mid-infrared images implies that massive stars are formed in a short period of time (less than a few million years) as the spiral wave passes through the disk. Consequently, models that include extended periods of star formation will not resolve the problem caused by the absence of a detectable lag between the $\mathrm{X}$-ray emission and $\mathrm{H} \alpha$ emission.

Visually inspecting the images in Figure 1, there does not appear to be any such lag between the diffuse X-ray and the $\mathrm{H} \alpha$ or mid-IR emission in any of the images. The diffuse $\mathrm{X}$-ray emission images have been smoothed to enhance the faint diffuse features; however, this should not affect the location of the diffuse emission. When we ran the adaptive smoothing routine, we restricted the maximum smoothing scale to be $20^{\prime \prime}$. The smoothing would not have moved the centroid of the X-ray emission but only degraded the spatial resolution. We note that we saw no obvious visual evidence for fine structure in the diffuse X-ray emission in the unsmoothed maps. Higher signal-to-noise ratio images would probably reveal more structure in the diffuse component, but we still expect that the hot gas is distributed over fairly large regions.

In Figure 4 we show diffuse X-ray contours on top of grayscale images of the $\mathrm{H} \alpha$ and mid-IR emission for each of the three galaxies M51, M83, and NGC 3184 that show strong, open spiral structure in all three wavelengths. In M83 and M51 it appears that the X-ray emission in some regions extend to somewhat larger radii than the mid-IR emission. However, there is no such extension seen in NGC 3184, where the X-ray contours are nearly centered on the regions of star formation.

While there is some difference between the distribution of hot gas and that seen in $\mathrm{H} \alpha$, mid-IR, and diffuse X-ray emission, there is no clear lag between the X-ray emission and that tracing massive star formation. The only major difference is that the X-ray emission is more diffuse than the $\mathrm{H} \alpha$ emission. This would be expected if the X-ray emission arose from a somewhat larger scale height above the plane of the galaxy than the $\mathrm{H}$ II regions.

Population synthesis models for the X-ray luminosity are also dependent on an efficiency factor $\epsilon$ that represents the fraction of mechanical energy that heats the ambient gas to $\mathrm{X}$-ray-emitting temperatures. The adiabatic phase of expan- sion depends on the density of the ambient medium and would be shorter in a denser medium. However, the X-ray luminosity could be higher in such a medium because the density of shocked material would be higher. Supernovae that occur in denser regions probably produce more X-ray emission (Smith $\&$ Cox 2001). Our observations suggest that $\epsilon$ is dependent on environment and is higher near sites of massive star formation than in lower density inner-arm regions. Because the X-ray emission along the spiral arms is quite narrow, we infer that supernovae from interarm regions must produce less soft X-ray emission than those that explode near the sites of massive star formation.

\subsection{Comparison of Diffuse X-Ray Emission and Star-forming Regions with Galactic Fountain Models}

The narrowness of the X-ray emission associated with the strong spiral arms can be used to place a limit on the vertical scale height of coronal gas. The vertical scale height is likely to be smaller than the width of the features as seen on the sky. The width of the features seen in X-ray is typically less than $50^{\prime \prime}$ wide, implying that the vertical scale height of the hot gas is likely to be less than $1-2 \mathrm{kpc}$ for these galaxies. Since this emission is located at $5-10 \mathrm{kpc}$ from the galaxy centers, this implies that the aspect ratio of the coronal emission $h / r \lesssim 1 / 4$. The X-ray gas at large radii in these galaxies is likely to be in a fairly thin layer. While the hot gas is likely to be above the $\mathrm{H}$ I disk, it is not likely to be more than a few kpc above the plane of the galaxy. We see no evidence for a bright X-ray halo component at large radii in these galaxies. Even in the starburst galaxy M83, the fact that the X-ray emission is associated with the spiral arms in the outer part of the galaxy suggests that it is tied to the disk and not part of the halo. This would suggest that a galactic fountain can operate at fairly high rates of star formation.

There is, however, a change in morphology seen in the $\mathrm{X}$-ray emission from disk emission associated with spiral structure in the outer region to a more nearly axisymmetric distribution in the center of these galaxies. The edge-on galaxies atlas by Strickland et al. (2004a) shows that large-scale outflows are primarily a phenomenon restricted to the central few kiloparsecs of galaxies. This suggests that many galaxies 
undergo a transition at some radius from a low scale height hot gas disk, associated with a "galactic fountain," to a more spherical distribution that might be associated with an outflow. At smaller radii the angular rotation rate is higher, so star formation associated with spiral arms will heat the ISM more frequently. If the gas cannot cool before the next spiral wave passes, by then each wave additively inputs energy into the hot component of the ISM. If this scenario is correct, then the radius at which the galaxy undergoes a transition in X-ray morphology would depend on the angular rotation rate and on the cooling time of the hot gas. Because the angular rotation rate is dependent on the mass distribution (and bulge), this could in part explain the weak correlation seen between the central X-ray and bulge surface brightnesses (Fig. 3a). Alternatively, the hot gas from stellar winds and Type I supernovae associated with an old bulge stellar population (accounting for the X-ray emission in early type galaxies; see, e.g., Brown \& Bregman 1998) could provide a reservoir of hot gas that might facilitate the driving of a superwind.

\section{SUMMARY AND DISCUSSION}

In this paper we have carried out a study of the diffuse soft X-ray emission in a sample of nearby low- and moderateinclination spiral galaxies. By using the high angular resolution possible with Chandra, we were able to separate between the diffuse component and that associated with point sources. We have compared the morphology of the diffuse emission with that at other wavelengths, finding the closest correspondence between the diffuse emission and indicators of massive star formation such as $\mathrm{H} \alpha$ emission or mid-infrared emission. In the spiral arms, the $0.1-2 \mathrm{keV} X$-ray energy flux is correlated with that in the mid-infrared tracing recent star formation and is about 5 orders of magnitude below that in emitted in the far-infrared.

The outer regions of these galaxies show that X-ray emission is closely associated with star formation lying in linear features following the spiral arms. Most of the galaxies also exhibit diffuse X-ray emission in the centers; however, the morphology of this component is approximately radially symmetric. The level of central X-ray emission is correlated with that seen in the mid-infrared and that from bulge stars in the near-infrared, although the correlation between the X-ray and mid-IR is not as tight as that seen in the spiral arms. It is likely that more than one process is contributing to the level of diffuse X-ray emission in these galaxy centers.

The narrow size of the X-ray emission in the spiral arms and estimated emission measures imply that the volume filling factor $f_{v} \lesssim 0.01$ and the scale height $h$ is less than a few kiloparsecs. Consequently, the X-ray gas in the spiral arms is probably associated with a galactic fountain and not part of a superwind. By a similar argument we reach the opposite conclusion for the hot gas in these galaxy centers, suspecting that the scale heights are larger than a few kiloparsecs and the filling factors are larger. The X-ray-emitting gas in these galaxy centers could be associated with galactic scale winds originating from the galaxy nucleus.

Contrary to the predictions of simple population synthesis models, the X-ray emission in the spiral arms does not lag the mid-IR or $\mathrm{H} \alpha$ emission. The narrowness of the X-ray emission implies that the X-ray gas produced in the arms must cool off quickly enough that it is not detected interarm, and that $\mathrm{X}$-ray gas is not efficiently produced outside of spiral arms. This suggests that the fraction of mechanical energy from supernovae that heats the ambient gas to X-ray-emitting temperatures is dependent on environment and is higher near the sites of massive star formation.

In this paper we have compared the X-ray morphology to tracers of current star formation. However, future work could probe the dependence of X-ray luminosity and morphology on the past star formation history by comparing these observations to optical and near-infrared spectroscopic observations. Future studies could also probe the relation between the LINER classification and the X-ray properties of galaxies.

We acknowledge helpful discussions with Margaret Drenner, Alaina Henry, Don Garnett, Dave Strickland, Chip Kobulnicky, Kip Kuntz, Jessica Almog, and Richard Mushotsky. We are grateful to Tony Wong for providing us with $\mathrm{H} \alpha$ images. We acknowledge support from the REU program NSF grant PHY-0242483 and Chandra GO grant GO0-1146X. This research has made use of the NASA/IPAC Extragalactic Database (NED), which is operated by the Jet Propulsion Laboratory, California Institute of Technology, under contract with the National Aeronautics and Space Administration.
Bendo, G. J., et al. 2002, AJ, 123, 3067

Bregman, J. N. 1980, ApJ, 236, 577

Bregman, J. N., \& Houck, J. C. 1997, ApJ, 485, 159

Brown, B. A., \& Bregman, J. N. 1998, ApJ, 495, L75

Cervino, M., Mas-Hesse, J. M., \& Kunth, D. 2002, A\&A, 392, 19

Chevalier, R. A., Clegg, A. W. 1985, Nature, 317, 44

Cui, W., Sanders, W. T., McCammon, D., Snowden, S. L., \& Womble, D. S. 1996, ApJ, 468, 102

de Avillez, M. A. 2000, Ap\&SS, 272, 23

Edmunds, M. G. 1990, MNRAS, 246, 678

Ehle, M., Pietsch, W., \& Beck, R. 1995, A\&A, 295, 289

Eracleous, M., Shields, J. C., Chartas, G., \& Moran, E. C. 2002, ApJ, 565, 108

Fabbiano, G., Kim, D.-W., \& Trinchieri, G. 1992, ApJS, 80, 531

Fraternali, F., Cappi, M., Sancisi, R., \& Oosterloo, T. 2002, ApJ, 578, 109

Gonzalez, R. A., \& Graham, J. R. 1996, ApJ, 460, 651

Heckman, T. M., Armus, L., \& Miley, G. K. 1990, ApJS, 74, 833

Helfer, T. T., Thornley, M. D., Regan, M. W., Wong, T., Sheth, K., Vogel, S. N., Blitz, L., \& Bock, D. C.-J. 2003, ApJS, 145, 259

Henry, A. L., Quillen, A. C., \& Guttermuth, R. 2004, AJ, in press

Kennicutt, R. C., Jr. 1998, ARA\&A, 36, 189

Koopmann, R. A., Kenney, J. D. P., \& Young, J. 2001, ApJS, 135, 125

\section{REFERENCES}

Kuntz, K. D., Snowden, S. L., Pence, W. D., \& Mukai, K. 2003, ApJ, 588,264

Larsen, S. S., \& Richtler, T. 1999, A\&A, 345, 59

Leitherer, C., \& Heckman, T. M. 1995, ApJS, 96, 9

Liedahl, D. A., Osterheld, A. L., \& Goldstein, W. H. 1995, ApJ, 438, L115

Martin, C. L., Kobulnicky, H. A., \& Heckman, T. M. 2002, ApJ, 574, 663

Mewe, R., Gronenschild, E. H. B. M., \& van den Oord, G. H. J. 1985, A\&AS, 62, 197

Mewe, R., Lemen, J. R., \& van den Oord, G. H. J. 1986, A\&AS, 65, 511

Norman, C. A., \& Ikeuchi, S. 1989, ApJ, 345, 372

Read, A. M., \& Ponman, T. J. 2001, MNRAS, 328, 127

Read, A. M., Ponman, T. J., \& Strickland, D. K. 1997, MNRAS, 286, 626

Roussel, H., et al. 2001, A\&A, 369, 473

Smith, B. J. 1998, ApJ, 500, 181

Smith, R. K., \& Cox, D. P. 2001, ApJS, 134, 283

Snowden, S. L., \& Pietsch, W. 1995, ApJ, 452, 627

Spinoglio, L., Malkan, M. A., Rush, B., Carrasco, L., \& Recillas-Cruz, E. 1995, ApJ, 453, 616

Strickland, D. K., Heckman, T. M., Colbert, E. J. M., Hoopes, C. G., \& Weaver,

K. A. 2004a, ApJS, 151, 193 2004b, ApJ, 606, 829 
Strickland, D. K., Heckman, T. M., Weaver, K. A., \& Dahlem, M. 2000, AJ, 120, 2965

Thim, F., Tammann, G. A., Saha, A., Dolphin, A., Sandage, A., Tolstoy, E., \& Labhardt, L. 2003, ApJ, 590, 256

Tully, R. B. 1988, Nearby Galaxies Catalog (New York: Cambridge Univ. Press)
Wang, Q. D., Immler, S., Walterbos, R., Lauroesch, J. T., \& Breitschwerdt, D 2001, ApJ, 555, L99

Wong, T, \& Blitz, L. 2002, ApJ, 569, 157

Young, A. J., Wilson, A. S., \& Shopbell, P. L. 2001, ApJ, 556, 6 